\title{
A New Conception of Measurement Uncertainty Calculation
}

\author{
J. JAKUBIEC* \\ Silesian University of Technology, Faculty of Electrical Engineering \\ Institute of Metrology, Electronics and Automation, Akademicka 10, 44-100 Gliwice, Poland
}

\begin{abstract}
The paper presents a conception of uncertainty calculation of a result obtained in a direct measurement realized in conditions described by random errors. The conception basis on the error definition being an effect of analysis of a quantization process and, first of all, it permits to determine uncertainty of a single measurement result in measuring and control systems processing signals varying in time. Division of the errors into two types A and B permits elaboration of such a procedure which enables uncertainty calculation for an average value of a series of measurements in the way close to this one proposed by GUM and widely discussed in last years. Theoretical considerations are illustrated by examples showing practical properties of the presented uncertainty calculation procedures.
\end{abstract}

DOI: $10.12693 /$ APhysPolA.124.436

PACS: 06.20.F-, 03.65.Ta, 06.20.-f

\section{Introduction}

The formula commonly used for uncertainty evaluation of measurement results is based on the following equation:

$$
u^{2}(y)=\sum_{i=1}^{N}\left(\frac{\partial f}{\partial x_{i}}\right)^{2} u^{2}\left(x_{i}\right)
$$

taken from GUM [1] which is treated in metrology as a container of fundamental rules dealing with inaccuracy of measurement data. Equation (1) is called in it as "the law of uncertainty propagation" and determines relations between variations of quantities described in probabilistic categories. Quantities $x_{i}, i=1,2, \ldots, N$, are measured directly and then, on the base of its estimates, a value of the quantity $y$ is calculated with assumption that a relation between these quantities is known as the function which generally can be written as

$$
y=f\left(x_{1}, x_{2}, \ldots, x_{N}\right) .
$$

Variances $u^{2}\left(x_{i}\right), i=1,2, \ldots, N$, in Eq. (1) are defined as squares of suitable so-called "standard uncertainties" which are in fact standard deviations (or their estimates) of quantities measured directly. Variance $u^{2}(y)$ is treated as a square of standard uncertainty $u(y)$ of the quantity $y$ measured indirectly.

Equation (1) can be also used to determine standard deviation (standard uncertainty) of a quantity measured directly [1]. In this case, all differentials in (1) are equal to 1 , therefore, it may be written in the form:

$$
u^{2}=u_{\mathrm{A}}^{2}+u_{\mathrm{B} 1}^{2}+u_{\mathrm{B} 2}^{2}+\ldots+u_{\mathrm{B} J}^{2},
$$

where the uncertainties have been divided into two categories. Uncertainty $u_{\mathrm{A}}$, named as type $\mathrm{A}$, is calculated statistically as a parameter of a series of measurement results. The uncertainties of type B: $u_{\mathrm{B} 1}, u_{\mathrm{B} 2}, \ldots, u_{\mathrm{B} J}$ are determined on the base of knowledge about measurement conditions.

\footnotetext{
*e-mail: jerzy.jakubiec@polsl.pl
}

The fundamental problem, dealing with the formulae applied for uncertainty calculation obtained on the basis of (3), consists in lack of formal agreement between its elements. One should notice that the considered uncertainties have to be seen as standard deviations of the same kind random quantities influenced on inaccuracy of a direct measurement. Taking into account the fact that type A uncertainty is calculated on the basis of a series of measurement results, one cannot point any other series which is obtained from the same measurement experiment. Therefore, the uncertainty $u_{\mathrm{A}}$ cannot be combined with any other uncertainty.

One may point some more weakness of the uncertainty calculation procedure based on GUM [1]. The most essential one results from the fact that this procedure needs a series of measurement results to determine uncertainty of type A, thus, it cannot be used for uncertainty calculation of a single measurement result. In many situations, especially happening in industrial measuring and control systems [2-5], measured quantities are varying in time and there are no possibilities to obtain more measurement results than one.

Let us analyze (3) once more. The basic question is: which assumptions should be taken in order to obtain formal acceptance of this equation? It is obvious that variances (squares of standard deviations or standard uncertainties) can be added if they are parameters describing some uncorrelated random quantities. In the discussed situation, these quantities have to be treated as "other quantities weighted according to how the measurement result varies with changes in these quantities" [1], which means that they are agents influencing on accuracy of the measurement result. From the mathematical point of view, such agents are considered as random errors [6]. Therefore, one can say in conclusion that the variances, added accordingly with (3), are parameters of suitable random errors which describe influences of factors essential for inaccuracy of the result measured directly [7].

Taking into account above, the error equation in the form 


$$
e=e_{\mathrm{A}}+e_{\mathrm{B} 1}+e_{\mathrm{B} 2}+\ldots+e_{\mathrm{B} J}
$$

can be treated as a formal basis of (3). According to (4), the realization of the total (combined) random error $e$ is the sum of realizations of the uncorrelated random partial errors denoted as $e_{\mathrm{A}}, e_{\mathrm{B} 1}, \ldots, e_{\mathrm{B} J}$. In this case, the error variances can be summed as it is described by (3).

One should notice that the partial errors in (4) have the same indexes as the variances in (3) because division of the errors into two categories $\mathrm{A}$ and $\mathrm{B}$, appropriate to the uncertainties A and B [1], is useful in some situations occurring in practice. Let us take then that the error of type A is such a kind of an error, probability distribution of which is determined by using statistical means while descriptions of the errors of type $B$ are obtained in the other ways "based on the degree of belief that an event (i.e. realization of the error) will occur" [1].

Having given variation of the error, one can add it to the variations of the other errors accordingly with (3) and extract the square root which finally results in obtaining the standard deviation of the total error. As a rule, the next operation consists in calculation of the expanded uncertainty, which is performed as multiplication of the standard deviation by the coverage factor [1]. This factor depends on distribution of the total error which means that (3) in its "clear" form may be used for the expanded uncertainty evaluation only if the total error can be described by the normal distribution, i.e. the central limit theorem [8] is compulsory during combining the partial errors. In other cases Eq. (3) is useless.

From these beginning considerations, one can draw the conclusion that modern metrology needs a new look to the problem of uncertainty calculation of a measurement result. Main part of the paper is devoted to presentation of the conception which permits to solve this problem for measurements performed both in laboratories and in industrial measuring systems. The base of this conception is a measurement error definition.

\section{Measurement error in description of a measured result}

To obtain the measurement error definition, one can analyse the case when a single measurement is realized. This case is typical for measuring systems [4, 5], input signals of which usually vary in time. Therefore, every measurement has to be performed in two stages: at first, the signal is sampled at the precisely determined moment and, after that, the sample is measured by an analog-to-digital (AD) converter. AD conversion is realized only one time, so one obtains only one measurement result for every sample and there is no possibility to repeat this action for the same instantaneous value of the signal.

From measurement point of view, AD conversion can be described as a direct comparison of the measured quantity with a sum of the same kind elementary measurement standards called quanta $[4,5]$. Every quantum has the same value which is much less than a working range of an $\mathrm{AD}$ converter. In voltage $\mathrm{AD}$ converters, the quanta sum is usually obtained by using a voltage divider built from resistors having the same values $R$ or connections $R$ with $2 R$ [5].

Generally, every measuring instrument working on the described above rule is called a quantizer. Its scheme, in the simplest form, is shown in Fig. 1a.

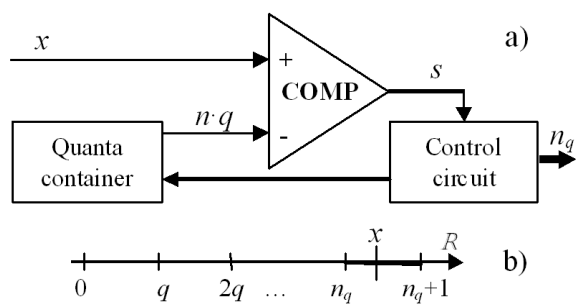

Fig. 1. (a) Basic scheme of a quantizer, (b) interpretation of a quantization result.

A quantization process can be performed in many different ways [5], but in every case the essence of this process consists in comparison of a value of the quantized quantity $x$ with the sum of $n$ quanta (every has the value $q$ ) delivered from the quanta container. Let us take that if $x-n q>0$ state of comparator COMP $s=1$, in the other case $s=0$. The control circuit increases number of quanta as long as $s=0$. Denoting the final number of quanta as $n_{q}+1$, where $n_{q}$ is the dimensionless indication of the quantizer being the number of quanta assigned to the value of measured quantity $x$, one can describe the quatization result as an interval in the way shown in Fig. 1b.

Accordingly with Fig. 1b, the quantization process consists in assignment of the selected interval, determined in real number set $R$, to the measured quantity $x$. Such an assignment is caused by the quantum character of the standard used in this process and let us to write that the true value of the measured quantity fulfils the inequality

$$
n_{q} q<x \leq\left(n_{q}+1\right) q,
$$

which describes the relation between value of measured quantity $x$ and number $n_{q}$ obtained as a dimensionless result of the quantization process.

For further considerations, one denotes

$$
\breve{x}=n_{q} q \text {, }
$$

where $\breve{x}$ is the dimensioned indication expressed in units of the measured quantity $x$ ( $\breve{x}$ can be called also a raw measurement result). After introducing expression (6) to $(5)$, one obtains

$$
\breve{x}<x \leq \breve{x}+q
$$

and then

$$
0<x-\breve{x} \leq q .
$$

Such a kind of difference as in (8), i.e. between a value of a measured quantity and a number obtained as a result of a measurement is commonly called a measurement error. In the described situation, measurement is performed by a quantizer and the error is defined as: 
$e_{\breve{x}}=x-\breve{x}$.

Equation (9) describes how much the measured value $x$ differs from the suitable indication $\breve{x}$ of a quantizer. Generally, this definition can be applied for every error of a measurement result.

After introducing the definition (9) to inequality (8), we have

$$
0<e_{\breve{x}} \leq q,
$$

which means that, in the considered situation, values of the quantization error change in limits from 0 to $q$. Interval (10) is not symmetrical. In this case, values of the error can be reduced (corrected) by subtracting its average value, equal to $0.5 q$ (see Fig. 2 ), in the following way:

$$
e_{\hat{x}}=e_{\breve{x}}-0.5 q \text {. }
$$

Coupling Eq. (11) and inequality (10) one obtains

$$
0<e_{\breve{x}}+0.5 q \leq q,
$$

which means that the corrected quantization error changes in symmetrical interval

$$
-0.5 q<e_{\hat{x}} \leq 0.5 q \text {. }
$$

The same result, as described by expression (13), can be obtained by replacing the indication $\breve{x}$ with its corrected value [5]:

$$
\hat{x}=\breve{x}+0.5 q \text {. }
$$

Accordingly with inequality (13), error $e_{\hat{x}}$ takes the least possible values. Taking it into account, corrected indication $\hat{x}$ can be treated as the best representation of the true value obtained from the measurement process and called an evaluation of a measured quantity.

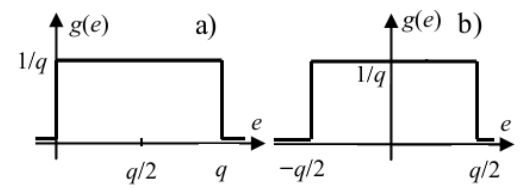

Fig. 2. Distributions of the quantization error: (a) with systematic component, (b) after correction of the systematic component.

It should be noticed that the correction may be done at the level of the dimensionless result $n_{q}$. After adding 0.5 to $n_{q}$ in the expression (6), one obtains the same effect as described by inequality (13) and Eq. (14).

Inequalities (10) and (13) determine only the limits of the quantization error. If one assumes that every measured value is as probable as the other values from the input range of the $\mathrm{AD}$ converter, the quantization error can be described in probabilistic categories. In this case the probability density functions of the error have the rectangular shapes shown in Fig. 2a and b, for Eqs. (10) and (13), respectively [5].

The distributions from Fig. 2a and $\mathrm{b}$ differ only with the expected values: the distribution from Fig. 2a has this value equal to $q / 2$ while this one from Fig. $2 \mathrm{~b}$ has the expected value equal to 0 . This property lets us to put the hypothesis that, from the uncertainty calculation procedure point of view, the measurement errors can be divided into two categories: systematic and random. Values of the systematic errors are known and may be eliminated by adding corrections to a measurement result, as it has been done in (14), or to the error (11). Such an elimination results in reducing the expected value of the random error to 0 . Therefore, the random errors are described in probabilistic categories, usually by using a probability density function (generally: by a distribution) with the zeroed expected value.

A source of a measurement error is generally characterized by a set of possible values which can be taken by this error in the determined measurement conditions. An error value set can be described in two ways: in probabilistic categories [6] or by a deterministic function [7]. A description of random properties of the error takes usually the form of a distribution which is a function of the error value. A deterministic description is used when it is possible to determine a quantity, which an error depends on, and a function that describes this dependence. Such a quantity is called an influence quantity.

There are two ways of using the deterministic function describing properties of an error. The first one consists in calculating the error value for the known value of the influence quantity. After that it is possible to use the calculated value as the correction in the way described above. The second way is applied if there is not possibility to know the value of the influence quantity but there are known limits of its changeability in measurement conditions. In this case, one can assume that the influence quantity changes uniformly within the given limits. It permits to determine frequency of the error value occurrence which can be interpreted as a description of the probability density function of the error [5].

The same error can be described in a deterministic way or in probabilistic categories dependently on measurement conditions. This property is illustrated by Example 1.

Example 1. Let assume that temperature $t$ varies from $t_{-}=10^{\circ} \mathrm{C}$ to $t_{+}=30^{\circ} \mathrm{C}$ and influences linearly on error $e$ as it is shown in Fig. 3a. Therefore, the influence function can be written as

$$
e(t)=a t+b,
$$

where

$$
\begin{aligned}
a & =\frac{e\left(t_{+}\right)-e\left(t_{-}\right)}{t_{+}-t_{-}}=\frac{3 \times 10^{-3}-1 \times 10^{-3}}{30-10} \\
& =0.1 \times 10^{-3} 1 / \mathrm{K}
\end{aligned}
$$

and

$b=e\left(t_{-}\right)-a \cdot t_{-}=1 \times 10^{-3}-0.1 \times 10^{-3} \times 10=0$.

Having temperature $t$ known, one can calculate $e(t)$ and use it as the correction. It is impossible if only limits $t_{-}$and $t_{+}$are known. In this case one can assume that all values of the temperature are of the same probability in interval $\left[t_{-}, t_{+}\right]$, which permits to determine probability density function $g(e)$ of error $e$ shown in Fig. 3b [5].

Distribution from Fig. $3 \mathrm{~b}$ is rectangular within limits: $e\left(t_{-}\right)=1 \times 10^{-3}$ and $e\left(t_{+}\right)=3 \times 10^{-3}$. In such a case, 


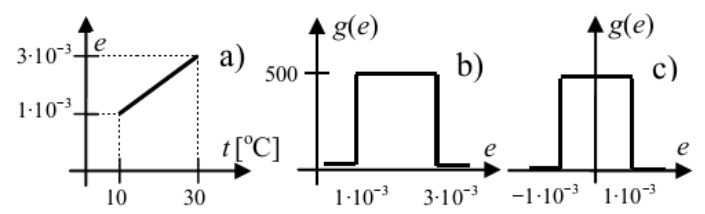

Fig. 3. (a) Deterministic description of exemplary error, (b) its probabilistic description with systematic component, (c) probabilistic description of the error after correction of the systematic component.

the expected value of error $e$ is equal to

$$
E(e)=\left(3 \times 10^{-3}-1 \times 10^{-3}\right) / 2=2 \times 10^{-3},
$$

which means that one can decrease every value of the error by subtracting correction

$$
c=E(e)=2 \times 10^{-3} \text {. }
$$

The same result can be obtained adding correction $c$ to the measurement result (see (14)).

After correction (see Fig. 3c), the error distribution becomes symmetrical in relation to the vertical axis and its changeability range is described by limits

$$
\begin{aligned}
& \text { lower : } e_{-}=e\left(t_{-}\right)-c=1 \times 10^{-3}-2 \times 10^{-3} \\
& \quad=-1 \times 10^{-3} \\
& \text { and higher : } e_{+}=e\left(t_{+}\right)-c=3 \times 10^{-3}-2 \times 10^{-3} \\
& \quad=1 \times 10^{-3} \text {. }
\end{aligned}
$$

\section{Uncertainty as a parameter of a measurement error value set}

From the presented above considerations, one can draw the conclusion that inaccuracy expression of a measurement result has to be based on mathematical description of error burdening this result. The error definition introduced as (9) is not taken arbitrary but it has the form resulting from analysis of the quantization process. Taking into account that properties of this process can be treated as representative for many measurement situations, the definition of a measurement error $e$ can be generally written in the form

$$
e=x-\hat{x},
$$

where $\hat{x}$ is an evaluation of a measurement result, the value of which is the most closer to the true value of a measured quantity. It means that performing a single measurement one cannot obtain a better value of a measured quantity than an evaluation. To the next considerations, one assumes that the evaluation is obtained by adding correction to the raw measuring result in this way that, after correction, the measurement error has expected value equal to 0 . Therefore, an error source burdening an evaluation is random and it is described by probability density functions with zeroed expected values.

Equation (15) is very important from uncertainty definition point of view. In GUM [1] the term "uncertainty of measurement" is generally interpreted as "doubt about the exactness or accuracy of the result of a measurement". Using probabilistic categories, this descriptive definition of the uncertainty can be written in the mathematical form as the expression:

$$
\operatorname{Pr}[|x-\hat{x}| \leq U]=p,
$$

where $\operatorname{Pr}$ means probability of such an event that absolute value of the difference between unknown true value of a measured quantity $x$ and its evaluation $\hat{x}$ is equal or less than uncertainty $U$. Accordingly with (16), the probability is equal to confidence level $p$, the value of which is typically taken as $p=0.95$.

Having given error definition (15), one can introduce it to expression (16) which after that takes the form

$$
\operatorname{Pr}[|e| \leq U]=p \text {. }
$$

If the error is described by symmetrical probability density function $g(e)$ with the expected value equal to 0 , one can write relation (17) as the functional

$$
\int_{-U}^{U} g(e) \mathrm{d} e=p .
$$

Example 2. Let us calculate uncertainty caused by the error described by probability density function shown in Fig. 3c. This function can be written as

$$
\begin{aligned}
& g(e)=a \quad \text { for } \quad e_{-} \leq e \leq e_{+}, \\
& g(e)=0 \quad \text { otherwise, }
\end{aligned}
$$

where the value of coefficient $a$ can be determined by using the equation resulting from the fact that function $g(e)$ has to satisfy the normalizing condition

$$
\int_{-\infty}^{\infty} g(e) \mathrm{d} e=1 .
$$

Taking values $e_{-}$and $e_{+}$from Example 1 and basing on (19) and (20), one obtains $a=500$.

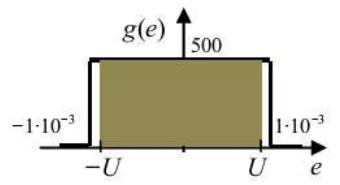

Fig. 4. Graphical interpretation of uncertainty $U$ calculated in Example 2.

Having given the error distribution as (19), one can determine value of the uncertainty from expression

$$
\int_{-U}^{U} a \mathrm{~d} e=0.95
$$

which has been obtained on the basis of (18). Taking into account that $a=500$, Eq. (21) is satisfied for $U=$ $0.95 \times 10^{-3}$. Graphical interpretation of uncertainty $U$ is given by using Fig. 4. It shows that uncertainty is a parameter of an error value set: it describes limits within which the area under the probability density function $g(e)$ is equal to $p=0.95$ ( $95 \%$ of the whole area under this function).

\section{Expression of a single measurement result as an interval}

The common approach to description of a measurement result consists in writing it as a numerical interval, 
the limits of which are determined by an uncertainty [1] Such a kind of description of measurement data is frequently used in the case when different mathematical means are applied for presentation these data in metrological categories [9-15].

Interpretation of the uncertainty given by Fig. 4 directly leads to the presentation of a single measurement result as a numerical interval. From (16) it results that probability of finding the true value of the measured quantity inside the interval described as

$$
|x-\hat{x}| \leq U
$$

is equal to $p$. Transforming inequality (22), one obtains expression:

$$
\hat{x}-U \leq x \leq \hat{x}+U
$$

describing limits of the interval which represents measuring quantity $x$ after its single measurement. The lower limit of the interval is

$$
\underline{x}=\hat{x}-U,
$$

while the upper one

$$
\bar{x}=\hat{x}+U \text {. }
$$

Therefore, the middle of the interval has the form [15]:

$$
\operatorname{mid}(x)=\frac{\bar{x}+\underline{x}}{2}=\frac{\hat{x}+U+(\hat{x}-U)}{2}=\hat{x}
$$

and the radius

$$
\operatorname{rad}(x)=\frac{\bar{x}-\underline{x}}{2}=\frac{\hat{x}+U-(\hat{x}-U)}{2}=U .
$$

The expressions presented above show that every single measurement result should be determined as the interval containing real numbers which means that measured quantity $x$ is transformed to interval $x$ in effect of a measurement process. The interval is built around the evaluation $\hat{x}$ being its middle (26) while interval radius is equal to uncertainty $U$. Therefore, the interval representing a measurement result with probability $p$ (it is called $95 \%$ coverage interval [16] for $p=0.95$ ) can be written as

$$
x=[\underline{x}, \bar{x}]=[\hat{x}-U, \hat{x}+U] .
$$

Processing measurement data by using algorithms [5] needs description of the interval in form (28) as two additive parts

$$
x=\hat{x}+[-U, U] .
$$

In form (29), the interval is composed of evaluation $\hat{x}$ and symmetrical interval $[-U, U]$ (its radius is equal to 0 ) which can be called an uncertainty interval. If the probability density function is non-symmetrical, the uncertainty interval is not symmetrical, too. Mathematical means that can be used in this case for determination of a measurement result as the interval have been described in $[5]$.

\section{Combined uncertainty}

In practice, every measurement result is burdened by many errors which means that the total uncertainty is a combination of partial uncertainties describing influences of the partial errors on inaccuracy of a measurement result. One can point four main ways of calculating the combined uncertainty. The first one consists in determination of standard deviation (standard uncertainty) accordingly with (3) and multiplication it by the coverage factor [16], value of which depends on both $p$ and shape of distribution of the combined error being sum (4) of the partial errors.

In the second way, all calculations are performed on uncertainty intervals determined for each partial error [17]. Possibility of taking into account correlations between partial errors is important property of this method [18].

The third way can be realized if combined error $e_{\mathrm{c}}$ is described as the sum of $N$ uncorrelated errors

$$
e_{\mathrm{c}}=\sum_{i=1}^{N} x_{i} .
$$

Knowing distributions of partial errors $e_{i}, i=1, \ldots$, $N$, one can determine the probability density function of the combined error making multi-step convolution accordingly with [4]:

$$
g_{\mathrm{c}}\left(e_{\mathrm{c}}\right)=g_{1}\left(e_{1}\right) * g_{2}\left(e_{2}\right) * \ldots * g_{N}\left(e_{N}\right),
$$

where $g_{i}\left(e_{i}\right)$ is probability density function of $i$-th error and $*$ is symbol of convolution. Hawing determined $g_{\mathrm{c}}\left(e_{\mathrm{c}}\right)$, one can use (18) to calculate the combined uncertainty.

The last procedure, most useful in practice and recommended by [16], is based on error model (30) and applied probabilistic simulation called Monte Carlo method. Except of making convolution (31), it applies (30) for summing up realizations of the partial errors, as it is shown in Example 3. The procedure is performed in many steps (in practice one uses about 100000 steps). Every step consists in summing values of partial errors taken from populations described by known probability density functions. The sum of realizations is located in the set containing values of the combined error. At the end of this procedure, the uncertainty is calculated on the base of this set by using functional (18).

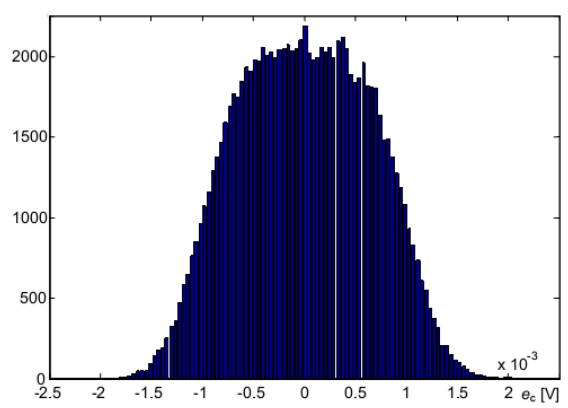

Fig. 5. Histogram of the combined error obtained in Example 3. Uncertainty calculated from this histogram for confidence level $p=0.95$ is $U=1.1 \times 10^{-3}$.

Example 3. Let assume that the combined error is the sum of two uncorrelated partial errors, i.e.: $e_{\mathrm{c}}=$ $e_{1}+e_{2}$. Error $e_{1}$ is described by rectangular probability density function shown in Fig. $3 \mathrm{~b}$ while the distribution of error $e_{2}$ is normal with standard deviation $\sigma=0.33 \times$ 
$10^{-3}$. The combined error calculated by using Monte Carlo method is shown in Fig. 5. More details about applications of this method to calculate uncertainties as parameters of errors can be found in [5].

\section{Errors of type A and B}

To calculate uncertainty of a measurement results accordingly with the described procedure, it is necessary to have given the probability density function of the total error. It means that if the total error is sum (30), distributions of all partial errors must be known. Therefore, descriptions of the errors have to be determined a priori, i.e. before a measurement realization. However, this requirement not always has to be fulfilled. One can point the situation when the error distribution may be determined after a measurement process - on the basis of a series of measurement results. In this case, it is necessary to divide partial errors into two categories: type $\mathrm{A}$ and type B, according to (4).

Taking into account considerations presented above and these ones from Sect. 1, one can qualify an error as type $\mathrm{B}$ if its distribution is known a priori while the distribution of type A error should be determined on the basis of a measurement series. Acceptance of this definition imposes specific requirements on properties of the errors of type $\mathrm{A}$ and $\mathrm{B}$ if one takes into account that both types of the error are random. At first, it means that all realizations of errors burdening measurement results in a series should be described as arising from one source, i.e. as the error of type A. At second, distribution of this kind of error should be described as normal because in practice only one parameter, i.e. a variance, is estimated on the basis of a series of measurements, which is caused by a limited number of results forming such a series.

One has to point an essential feature of errors if they are divided into these two categories. In the conditions, in which a measurement series is obtained, all errors of type $B$ take constant values because if not every change of some B type error is qualified to the set of realizations of A type error. Therefore, accordingly with (4), sum of B type errors can be written as

$$
e_{\mathrm{B} 1}+e_{\mathrm{B} 2}+\ldots+e_{\mathrm{B} J}=c,
$$

where $c$ is constant.

The described above feature is important because it enables obtaining an estimate of the variation of the $\mathrm{A}$ type error on the base of a series of measurement results. Let us notice that after introducing (4) and (32) to definition of a measurement error (15) it takes the form

$$
e_{\mathrm{A}}+c=x-\hat{x} \text {. }
$$

In Eq. (33), measured quantity $x$ has constant value because its invariability is the basic assumption taken for realization of a measurement series. Value of $c$ is also constant, therefore, from (33) it results that realizations of two random variables: error $e_{\mathrm{A}}$ and evaluation $\hat{x}$ differ in a constant value and have opposite signs. It means that both variables have the same variances. Taking this into account, one can calculate an estimate of the variance of error $e_{\mathrm{A}}$ on the base of a series of measurement results $\left\{\hat{x}_{i}, i=1, \ldots, n\right\}, n$ is the total number of measurements. The maximum likelihood estimator of the variance is determined as [8]:

$$
\bar{s}^{2}=\frac{1}{n-1} \sum_{i=1}^{n}\left(\hat{x}_{i}-\bar{x}\right)^{2},
$$

where $\bar{x}$ is the average value of the series described by expression

$$
\bar{x}=\frac{1}{n} \sum_{i=1}^{n} \hat{x}_{i} .
$$

The following example illustrates using a measurement series to determination of A type error distribution.

Example 4. Measurement process has delivered the series of 10 results: $\hat{x}_{i}=\{1.0003,0.9987,1.0007,1.0016$, $0.9993,1.0009,1.0013,0.9984,0.9986,1.0006\}$. On the basis of these results the average value, calculated accordingly with (35), is equal to

$$
\bar{x}=\frac{1}{10} \sum_{i=1}^{10} \hat{x}_{i}=\frac{10.0003}{10}=1.00003 .
$$

In this case, the variance estimator, described by (34), has the value

$$
\begin{aligned}
\bar{s}^{2} & =\frac{1}{10-1} \sum_{i=1}^{10}\left(\hat{x}_{i}-\bar{x}\right)^{2}=\frac{1.2742 \times 10^{-5}}{9} \\
& =1.4157 \times 10^{-6} .
\end{aligned}
$$

Error of type A has the same variance as the series of measurements. One assumes that all systematic errors have been corrected, thus, the expected value of this error is equal to 0 . In this situation, taking into account that the identification procedure may be used only for random errors with normal distribution, one can describe the distribution of A type error as normal $\mathcal{N}\left(0, \sigma_{\mathrm{A}}\right)$, where $\sigma_{\mathrm{A}}$ is standard deviation estimated as

$$
\sigma_{\mathrm{A}}=\sqrt{\bar{s}^{2}}=\sqrt{1.4157 \times 10^{-6}}=0.0012 .
$$

\section{Uncertainty of an average value of a measurement series}

Having determined distributions of A type error and errors of type B, one can calculate uncertainty of a single measurement result in the described way. However, in practice the same value of quantity is often measured many times which enables reducing inaccuracy if random error of type A dominates over the other errors (of type B). In this case, it is necessary to perform a series of measurements, on the basis of which average value (35) is calculated. Its uncertainty can be obtained in the way described in [5] with assumption that calculation of the average value is a kind of processing algorithm realized on a series of measurement results. This method is characterized below.

A processing algorithm operates on measurement data described by using a single measurement result model. This model, built on the base of error definition (9), has the form

$$
x=\hat{x}+e
$$

obtained with assumptions that all systematic errors have 
been corrected. Equation (36) means that an unknown true value of measured quantity $x$ is the sum of its evaluation $\hat{x}$ (obtained after correction of a single measurement result) and a realization of total (combined) error $e$. Generally, the combined error is given by sum (30) of partial errors which are described in probabilistic categories and uncorrelated as well as its probability density functions have expected values equal to zero.

Let us use model (36) in situation when a result of a measurement process is given as a series $\left\{\hat{x}_{i}, i=\right.$ $1, \ldots, n\}$ composed on $n$ evaluations of quantity $x$, which has a constant value during the process performed in the same measurement conditions. If every measurement result from this series is described by model (36), the average value (35) takes the form

$$
\frac{1}{n} \sum_{i=1}^{n} x_{i}=\frac{1}{n} \sum_{i=1}^{n}\left(\hat{x}_{i}+e_{i}\right)
$$

Taking into account that during the measurement experiment the true value of the measured quantity does not change, i.e. $x_{i}=x=$ const, $i=1, \ldots, n$, expression $(37)$ can be written as

$$
\begin{aligned}
x & =\frac{1}{n} \sum_{i=1}^{n}\left(\hat{x}_{i}+e_{i}\right)=\frac{1}{n} \sum_{i=1}^{n} \hat{x}_{i}+\frac{1}{n} \sum_{i=1}^{n} e_{i} \\
& =\bar{x}+\frac{1}{n} \sum_{i=1}^{n} e_{i},
\end{aligned}
$$

which means that if the evaluation of the measured quantity is given as average $\bar{x}(35)$, the total error of this average value is described by equation

$$
e_{\bar{x}}=\frac{1}{n} \sum_{i=1}^{n} e_{i}
$$

The measurement results forming the series are obtained in the same conditions which means that every result is burdened by errors of the same kind. Taking this into account and using description of errors type A and $\mathrm{B}$ in the form (4), one can write (39) as

$$
e_{\bar{x}}=\frac{1}{n} \sum_{i=1}^{n}\left[e_{\mathrm{A}}(i)+e_{\mathrm{B} 1}(i)+e_{\mathrm{B} 2}(i)+\ldots+e_{\mathrm{B} J}(i)\right] \text {. }
$$

As it has been discussed above, the series is measured with assumption that errors of type B have constant values. Therefore, for every error of type B, it takes place

$$
\frac{1}{n} \sum_{i=1}^{n}\left[e_{\mathrm{B} j}(i)\right]=\frac{1}{n} \sum_{i=1}^{n}\left[e_{\mathrm{B} j}\right]=e_{\mathrm{B} j}, \quad j=1, \ldots, J .
$$

In this case, Eq. (40) takes the form

$$
e_{\bar{x}}=\frac{1}{n} \sum_{i=1}^{n}\left[e_{\mathrm{A}}(i)\right]+e_{\mathrm{B} 1}+e_{\mathrm{B} 2}+\ldots+e_{\mathrm{B} J} .
$$

Error of type A is described in Eq. (42) as the sum of $n$ errors which burden measured results being elements of the series. Realizations of every partial error are taken from the same population of the random error $e_{\mathrm{A}}$, thus variation of this sum can be written as

$$
\sigma_{\mathrm{A} \bar{x}}^{2}=\sum_{i=1}^{n}\left[\frac{\sigma_{\mathrm{A}}^{2}}{n^{2}}\right]=\frac{n \sigma_{\mathrm{A}}^{2}}{n^{2}}=\frac{\sigma_{\mathrm{A}}^{2}}{n},
$$

where $\sigma_{\mathrm{A}}^{2}$ is variation of $\mathrm{A}$ type error. Taking into account the fact that this variation may be estimated from the measurement series by using (34), one can determine estimate of the standard deviation of the A type error for the average value (35) of the series on the base of equation

$$
\sigma_{\mathrm{A} \bar{x}}=\sqrt{\frac{\sigma_{\mathrm{A}}^{2}}{n}}=\sqrt{\frac{\bar{s}^{2}}{n}}=\sqrt{\frac{1}{n(n-1)} \sum_{i=1}^{n}\left(\hat{x}_{i}-\bar{x}\right)^{2}} .
$$

Equation (42) shows that errors of type B should be introduced if there is a need to expand measurement conditions in relation to these ones in which the series has been obtained. Errors of type B describe all additional factors, random properties of which appeared if influence quantities change their values. In such a case, basing on (42) and (44), one can calculate standard deviation of the total error as

$$
\sigma_{\bar{x}}=\sqrt{\sigma_{\mathrm{A} \bar{x}}^{2}+\sigma_{\mathrm{B} 1}^{2}+\sigma_{\mathrm{B} 2}^{2}+\ldots+\sigma_{\mathrm{B} J}^{2}},
$$

where $\sigma_{\mathrm{B} 1}, \sigma_{\mathrm{B} 2}, \ldots, \sigma_{\mathrm{B} J}$ are standard deviations of type $\mathrm{B}$ errors.

Equation (45) is usable for calculation of the expanded uncertainty if one disposes information that the combined error has the distribution close enough to the normal one. In this case, the uncertainty is obtained by multiplying standard deviation (45) (standard uncertainty) by the proper coverage factor suitable for taken value of the confidence level $p[16]$. Otherwise, one can use two ways. The first way consist in determination of the total error distribution by using convolution (31) or Monte Carlo method and then, using functional (18) to calculate the uncertainty. Application of the second way needs knowledge about values of expanded uncertainties of the partial errors. Having it, one can calculate the composed uncertainty using reductive interval arithmetic described in $[17,18]$.

\section{Basic procedures of uncertainty calculation}

Taking the presented considerations into account, one can point two basic procedures which can be used in practice to calculate uncertainty of a measurement result:

- Procedure 1 - applied if only a single measurement has been performed.

- Procedure 2 - the final measurement result is calculated as average value (35) on the basis of the measurement series.

Procedure 1. This procedure is realized in the following steps:

1. In the beginning, one should determine all partial error sources and describe them by using symmetrical probability density functions with zeroed expected values. In the case when expected value of 
the partial error differs from zero, it is needed introducing the proper correction in the way described in Sect. 2.

2. The presented procedure can be used only for uncorrelated errors (when they are correlated one can use procedure described in [16]), therefore, the next step consists in determination of the total error as the set of values obtained as sum (30) of realizations of the partial errors. The probability density function of the total error can be determined using convolution (31) but the simpler way is to determine the histogram of the error occurrence by using Monte Carlo method as it is shown in Example 3.

3. Having described the total error one can calculate uncertainty of a single measurement result solving functional (18) for taken confidence level $p$. The uncertainty value describes a priori inaccuracy of every single measurement result obtained in the conditions characterized by the selected errors.

4. The last step consists in writing the measured result as interval (28), limits of which are determined by the evaluation of the measured quantity and the calculated uncertainty.

Example 5. Let us take that conditions, in which a single measurement of voltage $x$ is performed, are characterized by two errors: $e_{1}$ with normal distribution $\mathcal{N}\left(0,1 \times 10^{-3}\right) \mathrm{V}$ and $e_{2}$ with rectangular distribution in limits [-0.001, 0.001] V. These errors have been determined before realization of measurements, therefore one calculate uncertainty a priori as the parameter describing inaccuracy of every single measurement. In this case, the total error $e_{\mathrm{c}}$ is the sum of two errors $e_{1}$ and $e_{2}$. Using Monte Carlo method, one obtains the histogram of the total error shown in Fig. 6.

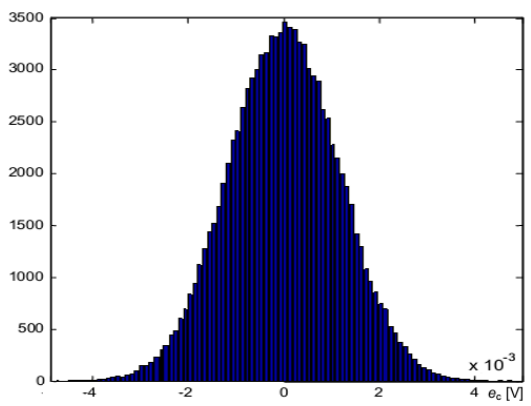

Fig. 6. Histogram of the combined error obtained in Example 5. Uncertainty calculated from this histogram for confidence level $p=0.95$ is $U=1.8 \times 10^{-3}$.

On the base of this histogram, the uncertainty calculated accordingly with (18) for confidence level $p=0.95$ has value

$$
U=1.8 \times 10^{-3} \mathrm{~V} .
$$

Let us assume now that a single measurement has been performed and the obtained value of the evaluation is for example: $\hat{x}=0.8577 \mathrm{~V}$. The measurement result can be written then as interval:

$$
\begin{aligned}
x & =0.8577+\left[-1.8 \times 10^{-3}, 1.8 \times 10^{-3}\right] \\
& =[0.8559,0.8595] \mathrm{V} .
\end{aligned}
$$

Procedure 1a. This procedure is performed in the same steps as procedure 1 with this difference that the error sources are divided into types A and B. Error of type $\mathrm{A}$ is determined on the basis of the series obtained from a measurement experiment performed especially to this aim. The variance of this error is estimated by using (34). Determination of distributions of the B type errors is realized in the same way as for procedure 1, i.e. by using knowledge about conditions of the measurement process which are characterized by influence quantities.

Example 6. Let us assume that the error model of a single measurement result of a voltage contains two errors: $e_{\mathrm{A}}$ of type $\mathrm{A}$ and $e_{\mathrm{B}}$ of type $\mathrm{B}$. Error of type $\mathrm{A}$ is identified on the basis of a series of measurements. Let us take that realization of the identification experiment has delivered the series of results taken in Example 4. The estimate of the standard deviation, calculated in Example 4 on the basis of this series, has the value: $\sigma_{\mathrm{A}}=0.0012 \mathrm{~V}$. Therefore, type A error is described by the normal distribution $\mathcal{N}\left(0,1.2 \times 10^{-3}\right) \mathrm{V}$. The error of type $\mathrm{B}$ describes such a component of inaccuracy which results from the fact that measurements are performed in temperature that influences on measured results but its value is not known. Taking that error of type B has properties described in Example 2, its distribution is rectangular in limits [-0.001, 0.001] $\mathrm{V}$. The histogram of the total error has been determined by using Monte Carlo method in the same way as in Example 3. On the base of this histogram, the uncertainty calculated accordingly with (18) for confidence level $p=0.95$ has value

$$
U=2.1 \times 10^{-3} \mathrm{~V} \text {. }
$$

If one assumes that the evaluation value of a single measurement is $\hat{x}=0.8577 \mathrm{~V}$, it can be written as interval

$$
\begin{aligned}
x & =0.8577+\left[-2.1 \times 10^{-3}, 2.1 \times 10^{-3}\right] \mathrm{V} \\
& =[0.8556,0.8598] \mathrm{V} .
\end{aligned}
$$

Procedure 2. It is realized as follows:

1. Having obtained a series of measurement results, at first one should calculate the average value of the measured quantity accordingly with (35).

2. The next step consists in estimation of the standard deviation of the type A error for the average value of the series on the base of (44) with assumption that it is of the normal distribution.

3. Distributions of the type B errors are determined on the base of knowledge about conditions of the measurement process.

4. In the next step, the probability density function of the total error is determined using convolution (31) or Monte Carlo method. 
5. Having described the total error, one performs uncertainty calculation of the average value of the series by using (18).

6 . The last step of the procedure consists in writing the measured result as the interval (28), the boundaries of which are determined by the evaluation of the measured quantity and the calculated uncertainty.

Example 7. The series of measurement results, taken in Example 4, has been obtained. The final result is determined in this case as average (35) of the series. The average is calculated in Example 4 and its value is

$$
\bar{x}=1.00003 \mathrm{~V} \text {. }
$$

Error of type A can be identified in this case on the basis of the same series of measurements as the average value, therefore, its standard deviation is estimated accordingly with (44) and has value

$$
\sigma_{\mathrm{A} \bar{x}}=\frac{0.0012}{\sqrt{10-1}} \mathrm{~V}=0.33 \times 10^{-3} \mathrm{~V} .
$$

The distribution of error of type $\mathrm{A}$ has to be assumed as normal, thus it is described as $\mathcal{N}\left(0,0.33 \times 10^{-3}\right) \mathrm{V}$. Error of type B has the same properties as this one in Example 6, i.e. its distribution is rectangular in limits $[-0.001,0.001] \mathrm{V}$. The histogram of the total error, determined by using Monte Carlo method, is the same as shown in Fig. 5 (the errors in Example 3 have the same distributions as in the presented example). On the basis of this histogram, the uncertainty calculated accordingly with (18) for confidence level $p=0.95$ has value

$$
U=1.1 \times 10^{-3} \mathrm{~V} \text {. }
$$

Therefore, the final result being the average value of the series can be written as interval

$$
\begin{aligned}
x & =1.00003+\left[-1.1 \times 10^{-3}, 1.1 \times 10^{-3}\right] \mathrm{V} \\
& =[0.9989,1.0011] \mathrm{V} .
\end{aligned}
$$

\section{Conclusions}

The presented conception of uncertainty calculation of a result measured directly bases on the definition of the measurement error. An uncertainty is determined as a parameter describing a set of the error values treated as realizations of a random quantity. Although the form of the error definition is the effect of the quantization process analysis, one can use this definition in every measurement situation. The reason of such a point of view results from the fact that in modern metrology one can notice the tendency to build standards basing on quantum phenomena. Moreover, every measurement instrument is characterized by its resolution which causes that number of possible measurement results is limited in the way close to this one occuring in the quantization process.
Using the error definition as a starting point of this conception enables obtaining the procedure of uncertainty calculation which is relatively simple and formally well grounded. The procedure is mainly dedicated to calculate uncertainty of a single measurement result which enables application of this procedure in measuring and control systems to obtain instantaneous values of signals varying in time. As it is shown in the paper, the procedure may be used for determining uncertainty of the average value of a series of measurements and, in general, for calculating uncertainty of algorithms [5] commonly used for measurement data processing. Division of the errors into separated types A and B permits to obtain the uncertainty calculation procedure close to this one proposed in GUM [1] and in its supplement [16].

\section{References}

[1] Guide to the Expression of Uncertainty in Measurement, ISO 1992, 1995.

[2] R. Kacker, K. Sommer, R. Kessel, Metrologia 44, 513 (2007).

[3] W. Bich, Metrologia 49, 702 (2012).

[4] J. Jakubiec, Metrol. Measur. Syst. 12, 406 (2004).

[5] J. Jakubiec, Errors and Uncertainties in Measuring and Control Systems, no. 176, SUT, Gliwice 2010, (in Polish).

[6] W.F. Fuller, Measurement Error Models, Wiley, New York 1987.

[7] A. Van der Veen, M.G. Cox, Metrologia 40, 45 (2003).

[8] A. Papoulis, Probability, Random Variables, and Stochastic Processes, Wiley, New York 1965.

[9] W. Jakubik, M. Urbanczyk, E. Maciak, T. Pustelny, Bull. Pol. Acad. Sci. Techn. Sci. 56, 133 (2008).

[10] W. Batko, P. Pawlik, Acta Phys. Pol. A 121, 152 (2012).

[11] T.R. Birdie, K.S. Surana, Reliable Comput. 4, 269 (1998).

[12] D.I. Doser, K.D. Crain, M. Baker, Reliable Comput. 4, 241 (1998).

[13] C. Tyszkiewicz, T. Pustelny, Opt. Appl. 34, 507 (2004).

[14] O. Kosheleva, V. Kreinovitch, Reliable Comput. 5, 81 (1999).

[15] A. Neumaier, Interval Methods for Systems of Equations, Cambridge University Press, Cambridge 1990.

[16] Guide to the Expression of Uncertainty in Measurement. Supplement 1. Numerical Methods for the Propagation of Distributions, ISO, 2004.

[17] J. Jakubiec, Application of Reductive Interval Arithmetic to Uncertainty Evaluation of Measurement Data Processing Algorithms, Monograph no. 26, SUT, Gliwice 2002.

[18] J. Jakubiec, Metrol. Measur. Syst. X, 137 (2003). 